\title{
Observation of a continuous interior crisis in the Hindmarsh-Rose neuron model
}

\author{
J. M. González-Miranda ${ }^{a}$ \\ Departamento de Física Fundamental, Universidad de Barcelona, Avenida Diagonal 647, \\ 08028 Barcelona, Spain
}

(Received 6 March 2003; accepted 5 June 2003; published 1 August 2003)

\begin{abstract}
Interior crises are understood as discontinuous changes of the size of a chaotic attractor that occur when an unstable periodic orbit collides with the chaotic attractor. We present here numerical evidence and theoretical reasoning which prove the existence of a chaos-chaos transition in which the change of the attractor size is sudden but continuous. This occurs in the Hindmarsh-Rose model of a neuron, at the transition point between the bursting and spiking dynamics, which are two different dynamic behaviors that this system is able to present. Moreover, besides the change in attractor size, other significant properties of the system undergoing the transitions do change in a relevant qualitative way. The mechanism for such transition is understood in terms of a simple one-dimensional map whose dynamics undergoes a crossover between two different universal behaviors. (C) 2003 American Institute of Physics. [DOI: 10.1063/1.1594851]
\end{abstract}

Nonlinear systems are usually able to display different dynamic behaviors depending on system parameters and external inputs. When these are slightly modified the system dynamics usually experiences little modifications, except when these changes occur in the vicinity of a critical point, in which case an abrupt qualitative change or transition in the dynamics occurs. An important example of these transitions is the case when the system changes its behavior from periodic to chaotic. This has been an object of major attention since the beginning of the study of chaos theory, and several types of transition, called routes to chaos, have been discovered. Chaos-chaos transitions in which the nature of the chaotic dynamics changes abruptly between two qualitatively different chaotic attractors have received less attention, but are equally important. In this article we present the observation of a chaos-chaos transition of a new type. This occurs in a system which belongs to a class which is of major importance in biological physics and in chemistry: a burstingspiking system. This kind of system is able to display two main types of dynamics: firing of spikes at more or less regular intervals, and bursting of trains of spikes interwoven with periods of quiescence. Neurons are one example of such systems for which the pattern of spiking is of great importance because it is believed that it codifies the information transmitted by the neuron. The transition between these two types of dynamics in a well established neuron model is studied here.

\section{INTRODUCTION}

Neurons can be seen as nonlinear dynamic systems where the relevant dynamic variable is the membrane potential that propagates along the axon. Experiments performed

\footnotetext{
${ }^{a)}$ Electronic mail: jgm@ffn.ub.es
}

on neurons subject to electrical stimulus result in the observation of two significant dynamic behaviors of the action potential. The simplest one consists in trains of sharp peaks known as spikes, while the other, which is more complicated, consists of bursts of spikes alternated by time lapses of quiescence. The nature of the dynamics in the two cases can be either periodic or chaotic. It is commonly believed that the information in the nervous system is encoded and transmitted by means of sequences of spikes, ${ }^{1}$ although it is controversial if the relevant observable is the mean frequency of the spike firing, or other statistical properties of the interspike intervals (see Refs. 2-4 and references therein). Moreover, spiking and bursting is also present in other types of electrically excitable cells, such as muscle fibers, ${ }^{5}$ in biochemically excitable cells, such as hepatocytes, ${ }^{6}$ and in chemical systems. ${ }^{7}$ This makes the study of spiking and bursting dynamics an object of major interest within the theory of nonlinear dynamics and chaos because of the special nature of the dynamics involved and because of its applications to neurobiology, other biophysical fields, chemistry and possibly other fields.

The electrical activity of a neuron is determined by the electrical properties of the axon membrane whose characterization requires at least two variables to describe the activity of the channels which exchange ions with the environment. These can be of one of two types: fast or slow. Moreover, there are also external inputs, which are given by electric currents injected in the neuron from the environment, other neurons, or the experimental setting. Hindmarsh and Rose ${ }^{8}$ have proposed a phenomenological model which takes this into account; it is a third order system of nonlinear ordinary differential equations which are able to display spiking or bursting dynamics depending on the system parameters which describe the state of the neuron and the external inputs. In appropriate ranges of parameters it has been found to be a realistic description of the electro-physics observed in experiments. For example, Rabinovich et al. ${ }^{9}$ have found 
that it provides a good description of the dynamics observed in experiments made with isolated neurons of the Pyloric control pattern generator of the California spiny lobster. Moreover, this model is being currently used in diverse theoretical and computational studies of the nonlinear dynamics of individual neurons ${ }^{10,11}$ as well as of ensembles of neurons. ${ }^{12,13}$

In this article we will study the transition between the bursting and the spiking dynamics in the chaotic regime of the Hindmarsh-Rose neuron model. We will show that the transition between these two types of behavior can be a chaos-chaos transition for which the attractor size changes suddenly but continuously as a system parameter is varied. The existence of this transition is of interest in neurobiology, because this provide a mechanism that allows rapid switching between different relevant neuronal behaviors. Then, the study of this transition may be useful to understand how the nervous system is able to give fast responses to external stimulus. ${ }^{14,15}$

From the point of view of chaos theory, this behavior presents certain resemblance with the chaos-chaos transition known as interior crisis that was discovered by Grebogi, Ott and Yorke. ${ }^{16}$ These authors observed discontinuous changes of attractor size caused by the collision of an unstable periodic orbit with a small sized attractor at certain critical parameter value. In contrast, the transition presented here, although very steep, is continuous; therefore, we propose to call it a continuous interior crisis. The mechanism for the continuous interior crisis is also different than the one governing the discontinuous crisis. ${ }^{16}$ By means of the study of an appropriate one-dimensional map we will show that the transition between the bursting and the spiking regimens can be seen as a continuous change between two universal types of nonlinear dynamics: the dynamics of the quadratic map, which models the spiking behavior, and the dynamics for the asymmetric tent map, which models the bursting behavior.

This article is then organized as follows. In Sec. II we present numerical results for the Hindmarsh-Rose model ${ }^{8}$ which show that this system is able to undergo a continuous interior crisis when the transition from bursting to spiking dynamics occurs. In Sec. III we will define a new one dimensional map whose behavior can smoothly change from bursting to spiking dynamics with the sharpness of the transition given by the properties of the map, providing in this way a mechanism for the continuous interior crisis. In Sec. IV we will discuss the above results both in terms of its interest to biophysics and of its relevance in the theory of dynamic systems and chaos, to finally end with a summary.

\section{CONTINUOUS CRISIS IN THE HINDMARSH-ROSE MODEL}

The Hindmarsh-Rose equations ${ }^{8}$ define a recognized model for the bursting-spiking dynamics of the membrane voltage, $x(t)$, observed in experiments made with single neurons. The equations of the model written in dimensionless form read

$$
\begin{aligned}
& \dot{x}=y+3 x^{2}-x^{3}-z+I, \\
& \dot{y}=1-5 x^{2}-y,
\end{aligned}
$$

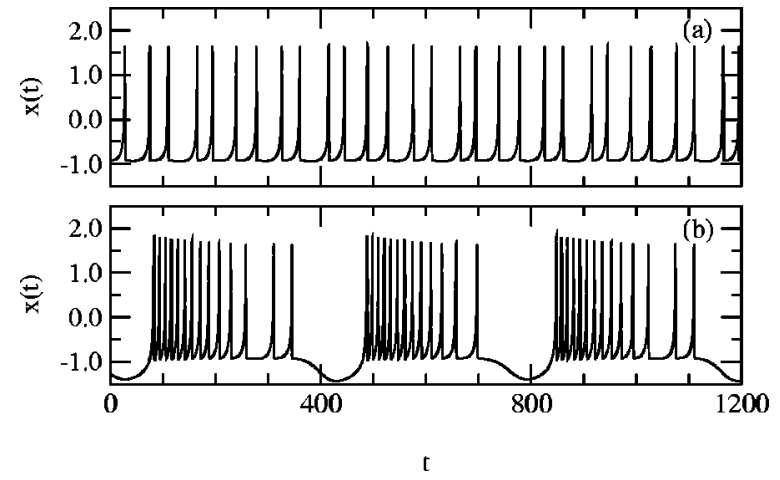

FIG. 1. Two types of dynamic behavior for the dynamics of the HindmarshRose model given by Eqs. (1)-(3) with $r=0.0021$ : (a) spiking dynamics at $I=3.31$, and (b) bursting dynamics at $I=3.28$.

$$
\dot{z}=-r\left[z-4\left(x+\frac{8}{5}\right)\right] .
$$

In these equations $y(t)$ and $z(t)$ are auxiliary variables describing, respectively, fast and slow transport processes across the membrane. The external current applied, I, and the internal state of the neuron, $r$, are the control parameters of the model used often. Rabinovich et al. ${ }^{9}$ have found that for $r \approx 0.0021$ this is a realistic description of the electrophysics of certain neurons axons. The spiking and bursting regimes displayed by this system are represented in Fig. 1. The spiking behavior, as shown in Fig. 1(a), is characterized by a oscillation of $x(t)$, which can be viewed as a series of peaks or spikes. The bursting behavior [Fig. 1(b)] is characterized by the alternation between two types of dynamics: one of spiking nature and the other with no oscillations, in which the systems appear to be at rest; when a nonoscillatory time interval ends with the first spike of a series we have a burst. The transition between these two types of dynamic behaviors when a control parameter is changed will be studied here.

For this nonlinear oscillator, working in the chaotic regime, we have observed that a small change of the value of $I$ (or $r$ ) in a proper critical region results in a large change of the attractor size, as well as in qualitative changes of other significant properties such as the statistics of the return times of $x(t)$. Although we will present here results mainly for $r$ $=0.0021$, we will note that similar behaviors can be observed for other sets of parameter, and for changing $r$ while $I$ is maintained fixed. At the above parameter values, this system is chaotic only within two disjoint intervals of values of $I,[3.135,3.150]$ and [3.222,3.319]. This last interval will be studied here. An elementary bifurcation analysis shows that the dynamics occurs around a single unstable fixed point having a spectrum of eigenvalues of the type $(-,+,+)$; i.e., a saddle. Numerical results for the two largest Lyapunov exponents, and the bifurcation diagram given from the consecutive maxima reached by $x(t), x_{M}$, are displayed in Figs. 2(a) and 2(b), respectively. They show how a chaotic attractor undergoes a sudden but continuous change around the value of $I_{C} \approx 3.2958$. This transition, as seen in the bifurcation diagram, bears a fast decrease of the size of the onedimensional attractor which describes the dynamics in the Poincare section, and a qualitative change in the nature of the 

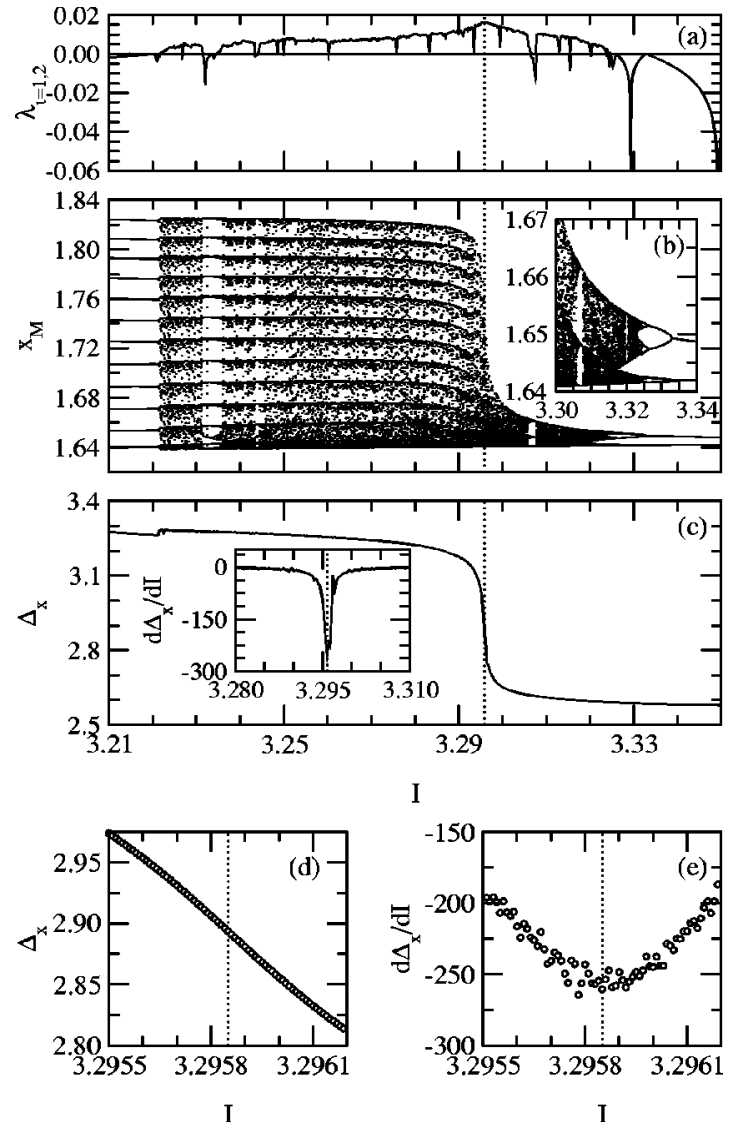

FIG. 2. Characterization of the dynamics of the Hindmarsh-Rose model given by Eqs. (1)-(3) with $r=0.0021$ as function of the external bias $I$ : (a) the two largest Lyapunov exponents, (b) bifurcation diagram given by the maxima of $x(t)$ (with a detailed view of the narrow part given in the inset), and (c) estimate of the size of the attractor as given by the range of variation of $x(t), \Delta_{x}(I)$ (with its derivative shown in the inset). The vertical dotted lines signal the critical value $I_{C}=3.29585$. (d) The function $\Delta_{x}(I)$, and (e) its derivative, both displayed in a narrow interval around the transition point.

dynamics as indicated in the inset where the well known portrait of dynamics of the quadratic map ${ }^{17,18}$ has emerged above $I_{C}$. The change in the dynamics around $I_{C}$ when $I$ increases does affect the size of the three dimensional attractor as well. This is demonstrated in Fig. 2(c) where the values of the range of variation of $x(t), \Delta x=x_{M a x}-x_{M i n}$, appear as a function of $I$ [similar results have also been obtained for $y(t)$ and $z(t)]$. There is a decrease in the size of the three-dimensional attractor along a curve which displays a sharp inflection point at $I_{C} \approx 3.29585$ which we identify as the critical point. The changes depicted in Figs. 2(b) and 2(c) occur within a narrow parameter interval, $\Delta I \approx 0.01$ (i.e., $\Delta I / I_{C} \approx 0.003$ ). This sudden continuous change is what we call here a continuous interior crisis. To get a more proper characterization of the transition a detailed study of $\Delta_{x}(I)$, and its derivative, has been performed in the close neighborhood of the transition point. To obtain reliable results, two averages have been performed to compute $\Delta_{x}(I)$ : for each choice of $I$, first a very large time average $\left(3 \times 10^{7}\right.$ time steps) was made for a given initial condition; then, the results obtained have been averaged over 240 different initial conditions. This provides a quite smooth result for $\Delta_{x}(I)$ around $I_{C}$ which indicates that the slope of this function at $I_{C}$ is
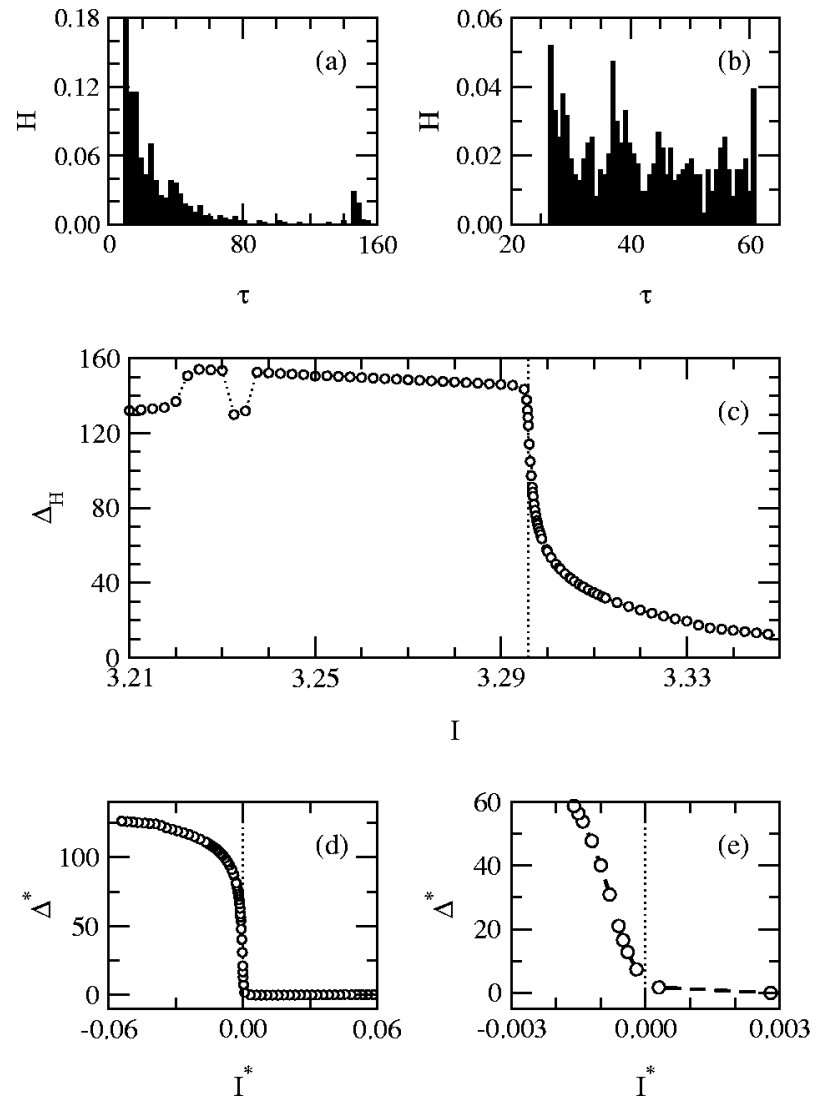

FIG. 3. Characterization of the bursting-spiking statistics of the Hindmarsh-Rose model with $r=0.0021$ as a function of the external bias $I$ : Examples of histograms in (a) the bursting regime $(I=3.28)$, and (b) the spiking regime $(I=3.31)$. (c) Dependence of the spread of the interspike intervals, $\Delta_{H}$, on $I$, (d) a scaled view of this dependency, and (e) a close-up to the transition region.

finite [Fig. 2(d)]. The numerical evaluation of the derivative of this function, $d \Delta_{x} / d I$, is very sensitive to small statistical errors because of the small interval between consecutive points which amplifies these errors when the numerical derivative is computed. However, we have been able to obtain, with the above statistics, the results in Fig. 2(e) which indicate a bounded value for $d \Delta_{x} / d I$ at the critical point. So we conclude that there is no singularity at the inflection point in this case.

The change of structure of the bifurcation diagram in Fig. 2(b) suggests that the crisis implies deep and significative variations in the properties of the dynamics before and after the transition. For this particular model, which is a representation of a real biophysical system, it is proper to study the dependence on $I$ of the dynamics of the peaks of the membrane voltage, $x(t)$. This is because it is assumed that the information carried along an axon by the membrane voltage is codified in the time intervals between successive maxima, ${ }^{1-4}$ i.e., in the interspike intervals, $\tau$. Because of this, it is common to characterize the dynamics of $x(t)$ by means of the interspike interval distribution function, $H(\tau)$. We have studied an estimate of $H(\tau)$ by computing interspike interval histograms as those shown in Figs. 3(a) and 3(b), where two plots of $H(\tau)$, each at a different side of the critical point, show how below the crisis these intervals are 
widespread along an asymmetric distribution, while above, the spread of the values of $\tau$ is much smaller and the distribution tends to be more symmetric. Above the transition we have the spiking regime where the time intervals between the maxima (spikes) are narrowly grouped around certain average values. Below the transition we have the bursting regime where spiking periods, with interspike intervals described by the short time part of the distribution, are separated by nonspiking intervals whose duration is given by the large time part of the distribution. The study of this chaos-chaos transition, as $I$ increases, in terms of $H(\tau)$ is given in the main body of Fig. 3(c), which shows the spread of the distribution, estimated as the difference between maximum and minimum values of the interspike intervals observed, $\Delta_{H}=\tau_{\operatorname{Max}}$ $-\tau_{M i n}$. The curve obtained shows indeed a continuous transition at $I_{C}$ between a region in which $\Delta_{H}$ remains nearly constant to another where it changes steadily. It is to be noted that there is a formal resemblance between the plot in Fig. 3 (c) and the curve of the order parameter in continuous phase transitions studied in condensed matter physics. This resemblance can be enhanced by means of the following trivial scaling transformation: $I^{*}=I_{C}-I, \quad \Delta^{*}=\left|\Delta_{H}-(a+b R)\right|$ with $a$ and $b$ given by a least squares fit to a straight line of the results for $\Delta_{H}(I)$ in the chaotic region with $I<I_{C}$. The transformation in $I$ just means to swap the above-below regimes, and the transformations in $\Delta_{H}$ mean to define an "order parameter" which is null in one of the two regimes and positive in the other. As seen in Fig. 3(d), the curves do look like those of a continuous phase transition, corresponding the ordered phase to the simply spiking attractor. However, we do not have the singular behavior characteristic of second order phase transitions, because the slope of $\Delta_{H}^{*}\left(I^{*}\right)$ stays bounded as we approach the transition point [Fig. 3(e)].

The phase diagram in the neighborhood of the transition point at $r_{C}=0.0021$ and $I_{C}=3.2958$ has been studied by means of the calculation of a segment of the line of critical points. This has been obtained from the derivative of $\Delta x$ with respect to $I$ at 20 different values of $r$ to obtain the values $I(r)$ where the extrema of $d \Delta / d t$ are. This provides the curve of critical points in the plane $I-r$ separating bursting from spiking dynamics which is displayed in Fig. 4(a). The sharpness of the transition along this critical line is measured by means of the absolute value of $d \Delta / d t$, presented in Fig. 4(b) which shows that it changes monotonically along the line of critical points being larger for smaller values of $r$. The largest Lyapunov exponent along this line remains almost constant [Fig. 4(c)] at a positive value $\left(\lambda_{1} \approx 0.014\right)$, which is an indication that the system stays chaotic along this curve.

\section{THE MECHANISMS FOR THE CONTINUOUS CRISIS}

We will use one-dimensional discrete maps of the form $x_{n+1}=f\left(x_{n}\right)$ which, being the simplest mathematical models able to display chaos, ${ }^{17,18}$ are good tools to understand the mechanism behind this transition. A well known model which holds the essence of many real chaotic systems is the quadratic map, which is given by

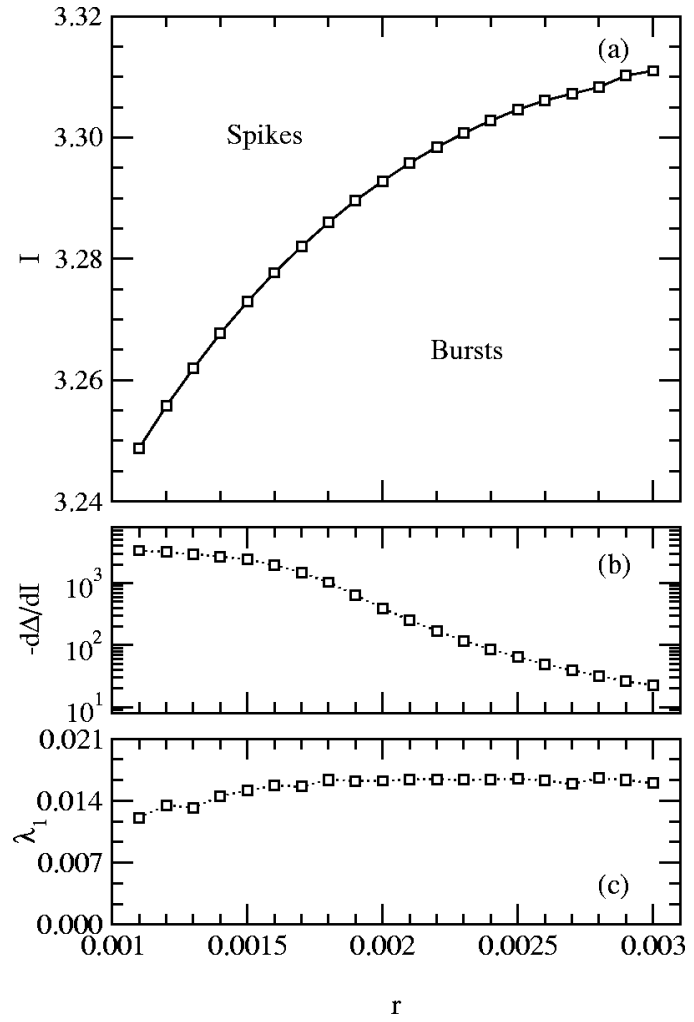

FIG. 4. Study of the phase diagram of the Hindmarsh-Rose model around the continuous crisis at $r=0.0021$. (a) Line of critical points between bursting and spiking dynamics in the plane $I-r$. (b) Absolute value of the derivative of the width of the distribution, and (c) the largest Lyapunov exponent, both along the line of critical points. In all these plots the squares indicate points obtained numerically and the lines are used to guide the eye.

$$
f(x)=2 C x+2 x^{2},
$$

with $C$ the control parameter. Its chaotic dynamics, as illustrated in Figs. 5(a), 5(c), 5(e), and 5(g), is characterized by irregular oscillations [Fig. 5(a)] caused by the motion around a fixed point close to the minima of $f(x)$ [Fig. 5(c)]. The dependence of the dynamic behavior on increasing $C$ has a characteristic bifurcation diagram [Fig. 5(e)] which results in the suppression of chaos by an inverse period doubling cascade. This behavior can also be followed by the Lyapunov exponent [Fig. 5(g)] which decreases with increasing $C$ along characteristic structures caused by periodic windows in the chaotic regime. We take this model as a paradigm of regular chaotic oscillations, i.e., of spiking behavior.

Another representative map displaying a different characteristic chaotic behavior is the tent map

$$
f(x)=\left\{\begin{array}{cc}
1-r x, & \text { if } x \leqslant 2 /(1+r), \\
x-1, & \text { if } x>2 /(1+r),
\end{array}\right.
$$

with $r$ a parameter, which takes positive values. Its dynamic behavior for large $r$ (i.e., for a highly asymmetric map) provides a prototype for bursting behavior, as show in Figs. $5(\mathrm{~b}), 5(\mathrm{~d}), 5(\mathrm{f})$, and $5(\mathrm{~h})$, where a representative point starting at large values of $x$ falls down along the second branch of the map towards the minimum of $f(x)$, and there the first 

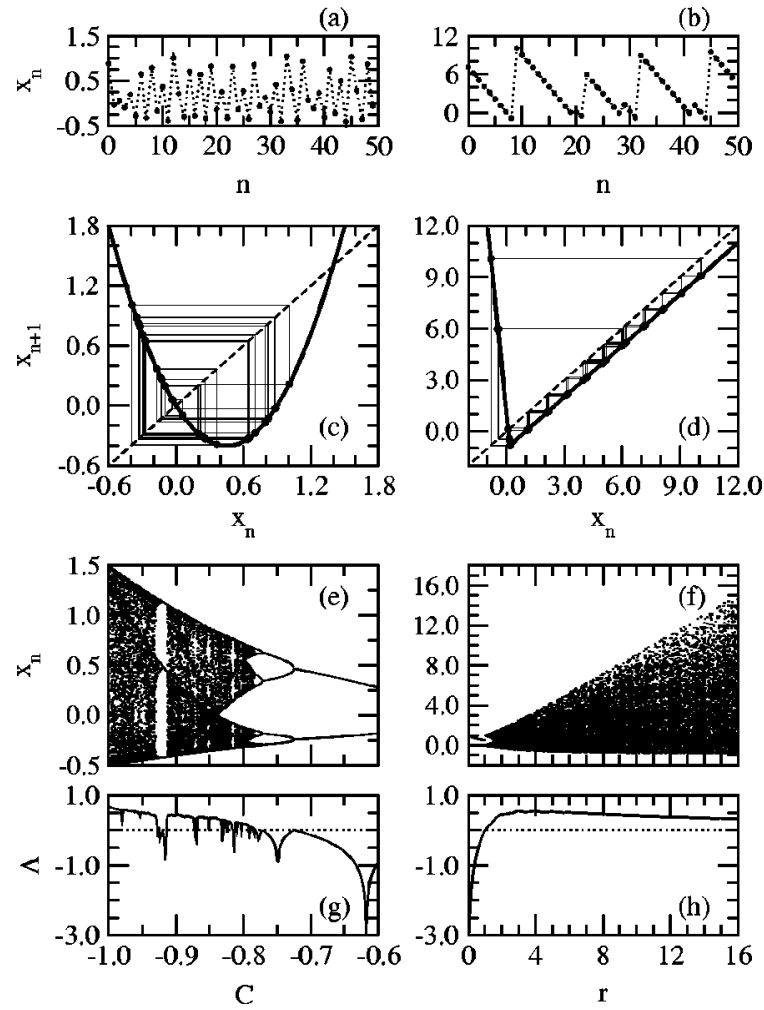

FIG. 5. Main features of the dynamics of the quadratic map (left) and the tent map (right). (a) and (b) Time series using dots for the iterates and dotted lines to guide the eye. (c) and (d) Plot of the map (thick line), iterated point (circles), tracks of the iterates (thin line), and the auxiliary $x_{n+1}=x_{n}$ line (dashed). (e) and (f) Bifurcation diagrams. (g) and (h) Lyapunov exponent (the $\Lambda=0$ line appears as a dotted line). The plots in (a) and (c) are for $C$ $=-0.90$ and $x_{0}=0.314$, and the plots in (b) and (d) are for $r=11.0$ and $x_{0}=0.141$ (in all cases to first $10^{4}$ points of the time series have been discarded).

branch sends it back to a large values of $x$ in an event which we identify as a burst. The bifurcation diagram [Fig. 5(f)] displays a band with a width that increases with the asymmetry of the map (i.e., with the height of the bursts) and displays little structure in the spatial distribution of phase space points. The Lyapunov exponent dependence on $r$ [Fig. $5(\mathrm{~h})]$ is almost flat and unstructured for sufficiently asymmetric maps. More details on the properties of each of these maps, which are examples of different universal behaviors for the dynamics of chaotic system, can be found in the literature. ${ }^{17,18}$ The transition from a sustained bursting regime to a simpler spiking regime can be modeled by a continuous transition between these two universal behaviors. For this aim we will use maps having the shape of an hyperbola so that the motion around the asymptotes is mainly of the type of the tent map, while the motion around the vertex is of the type of the quadratic map.

According to these ideas, we propose the following map that holds the essential features of real bursting-spiking systems, which we call the $2 \mathrm{H}$ map because it is made of two hyperbolas:

$$
f(x)=\left\{\begin{array}{cc}
0.10 /(x-\chi)+12, & \text { if } x \leqslant \bar{x} \\
\sigma / x+x-1, & \text { if } x>\bar{x} .
\end{array}\right.
$$
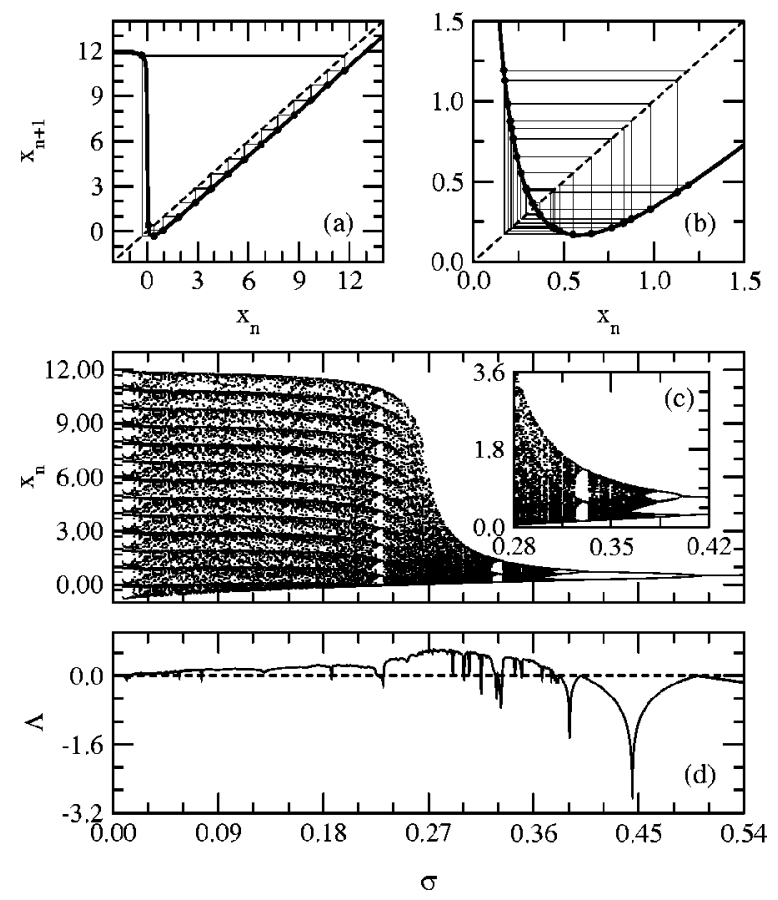

FIG. 6. Continuous interior crisis in the $2 \mathrm{H}$-map. (a) Dynamics of the type of the tent map for $\sigma=0.12$, and (b) of the type of the quadratic map for $\sigma=0.34$ and $x_{0}=0.141$, after $10^{4}$ iterations in the two cases [the different elements in these plots are to be identified as in Figs. 5(a) and 5(b)]. (c) Bifurcation diagram with an enlargement of the upper critical region show in the inset. (d) Lyapunov exponent (the $\Lambda=0$ line appears as a dashed line).

Here $\sigma$ is a control parameter, while $\bar{x}$ and $\chi$ are fixed by the following condition of continuity of the map:

$$
\begin{aligned}
& 0.10 /(\bar{x}-\chi)+12=12 R, \\
& \sigma / \bar{x}+\bar{x}-1=12 R,
\end{aligned}
$$

with $R \in(0,1)$ a parameter that is fixed at $R=0.5902164$ for most of the article. The branch for $x>\bar{x}$ has the shape of a hyperbola having its concavity up, the left asymptote is vertical while the right asymptote has slope equal to one, so providing a mechanisms for bursting. Around the minima the function is rounded, allowing spiking dynamics. The parameter $\sigma$ controls the concavity of the hyperbola and then the bursting intensity, allowing the transition between the two behaviors. The branch for $x \leqslant \bar{x}$ is a hyperbola having a left asymptote which is horizontal and a right asymptote which is vertical; this provides an additional control on the bursting activity which is needed to have the height of the bursts limited (i.e., a sustained bursting regime) as it occurs in real bursting-spiking systems.

The dynamic behavior for this map is presented in Fig. 6 . There is a sustained bursting regime that occurs at small values of $\sigma$ [Fig. 6(a)] which is similar to that of the tent map depicted in Figs. 5(b) and 5(d), and a spiking regime at larger values of $\sigma$ [Fig. 6(b)] similar to that of the quadratic map in Figs. 5(a) and 5(c). The bifurcation diagram and the Lyapunov exponent presented in Figs. 6(c) and 6(d) present a sustained bursting behavior for $\sigma \leqq 0.26$ and the characteristic structure of a quadratic map for $\sigma \geq 0.28$. There is a critical region around a critical point at $\sigma_{C} \approx 0.267$ in which the 

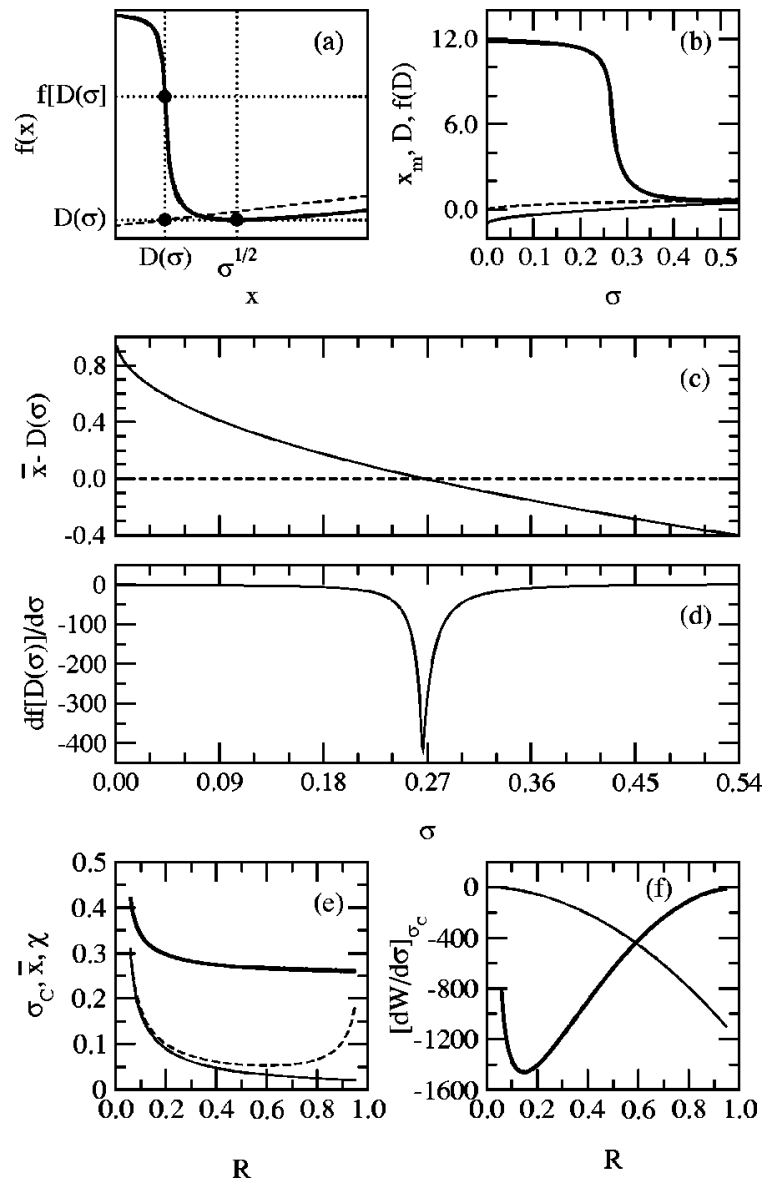

FIG. 7. Mechanism for the continuous interior crisis in the $2 \mathrm{H}$ map. (a) The function $f(x)$ with the relevant quantities written as labels in the axes, and the relevant points indicated by circles. The dashed line is $y=x$ (the particular curve plotted is for $\sigma=\sigma_{C}$ ). (b) Dependence of $x_{m}$ (dashed line), $D=f\left(x_{m}\right)$ (thin line), and $f(D)$ (thick line) on $\sigma$. (c) Difference between the abscissa of the inflexion point of the $2 \mathrm{H}$ map, $\bar{x}$, and the abscissa of the maximum reachable phase point, $D$, as a function of $\sigma$ (the condition $\bar{x}$ $-D=0$ has been plotted as a dashed line to guide the eye). (d) Plot of the derivative of $f[D(\sigma)]$ as a function of $\sigma$ (thick line). (e) Dependence on $R$ of the transition point, $\sigma_{C}$ (thick line), and the map parameters $\bar{x}$ (thin line) and $\chi$ (dashed line). (f) The derivative of the width of the attractor at the critical point computed from below (thick line) and from above (thin line) as a function of $R$.

size of the attractor decreases very fast, having an envelope which shows an inflection point, while the Lyapunov exponent reaches a broad maximum. This behavior is what we have called a continuous interior crisis.

The mechanism of the transition is as follows. There are three relevant quantities related to the function $f(x)$ which defines the 2H-map [Fig. 7(a)]: the abscissa of the minimum of $f(x), x_{m}=\sqrt{\sigma}$, and the images obtained applying $f(x)$ to $x_{m}$ once, $D(\sigma)=f\left(x_{m}\right)=2 \sqrt{\sigma}-1$, and twice, $f[D(\sigma)]$. $D(\sigma)$ is the minimum value of $f(x)$; then $f[D(\sigma)]$, the maximum value that a phase point, $x_{n}$, can reach under infinite iterations of the map. The functions $x_{m}(\sigma)$ and $D(\sigma)$ increase monotonously from $\sigma=0$, while $f[D(\sigma)]$ decreases monotonously [Fig. 7(b)]. Therefore, for $\sigma$ small $(\sigma \lesssim 0.26)$ the vertex of the map is very acute and the tent map behavior, with little contamination of the curved vertex, is dominant; moreover, the flat part at the left of the first branch of the map allows the height of the peaks to be stabilized against changes of $\sigma$. For $\sigma$ large $(\sigma \geqslant 0.28)$ the vertex of the map is broader and only the dynamics around the fixed point $x_{F}, \sigma / x_{F}+x_{F}-1=x_{F}$, is allowed giving rise to a quadratic map like behavior. The transition between these two regimes occurs at the value of $\sigma=\sigma_{C}$ at which the maximum value that the coordinate of a phase point, $x_{n}$, can reach, $f[D(\sigma)]$, equals the value of the abscissa of the inflection point of the map, $f(\bar{x})$, which is the point where the first branch of the map stops having an effect on the dynamics, thus signaling the end of the sustained bursting-spiking dynamics. The condition for the critical point is then

$$
D\left(\sigma_{C}\right)=\bar{x}
$$

This indeed occurs at $\sigma_{C} \approx 0.267$ as shown in Fig. 7(c). There is a narrow critical region around $\sigma_{C}$ because the highest value that $x_{n+1}=f\left(x_{n}\right)$ can reach for each $\sigma$ is given by $f[D(\sigma)]$ which, as shown in Fig. 7(d), is a continuous function which has zero slope in almost all of its range of definition except in a small region around $\sigma_{C}$ where the large slope of $f(x)$ around $\bar{x}$ causes large changes in $f[D(\sigma)]$ from tiny changes in $\sigma$; therefore, there is a sharp transition from bursting to spiking dynamics.

The study of the $2 \mathrm{H}$ map presented here has been made for a continuity condition [Eqs. (7) and (8)] with $R$ $=0.5902164$. The effect of changing $R$ on the parameters $\bar{x}$ and $\chi$ is small for intermediate values of $R(0.2<R<0.8)$, and so is the effect on $\sigma_{C}$ [Fig. 7(e)]. The change of $R$ has more notorious consequences when the width of the attractor of the $2 \mathrm{H}$ map, $W(\sigma)$, is considered. According to the discussion in the previous paragraph, this is given by $W(\sigma)$ $=f[D(\sigma)]-D(\sigma)$, as illustrated by the thin and thick lines in Fig. 7(b), which trace the envelopes of the attractor plotted in Fig. 6(c). $W(\sigma)$ is continuous and bounded at $\sigma_{C}$ because of the continuity and boundedness of $f$. However, its derivative at the critical point $[d W / d \sigma]_{\sigma=\sigma_{C}}$ may be discontinuous because when computed from $\sigma<\sigma_{C}$ is given by the lower branch of the $2 \mathrm{H}$ map, while when computed from $\sigma>\sigma_{C}$ is given by the upper branch. As illustrated in Fig. 7(f) this is indeed the case for all $R$, with the exception of $R$ $\approx 0.5902164$, which was the value chosen here with the purpose of studying a map in qualitative agreement with the results for the Hindmarsh-Rose model presented in Figs. 2(c) $-2(e)$.

It is to be noted that the overall critical behavior is not altered in an essential form when small modifications are made to the shape of the $2 \mathrm{H}$ map by modifying Eq. (6). This is illustrated in Fig. 8 for four such modifications. Although there are certain changes on the values of $\sigma$ at which there are periodic windows, on the structure of the spatial distribution of the map points, and on the steepness of the transition, in all cases, we observe the same general features described in the above paragraphs.

Finally, one has to note that the analogy between interior crisis and phase transitions suggested here is only formal, as one should expect from the different nature of the systems implied: deterministic for the crisis, and statistical for phase transitions. According to the above results this appears limited to the existence of discontinuous and continuous 

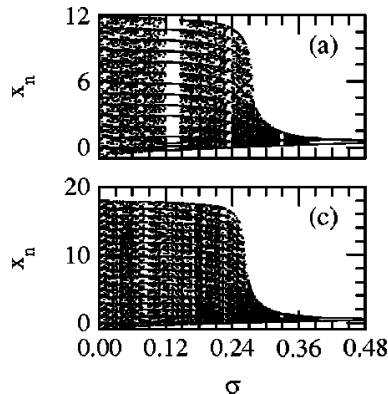
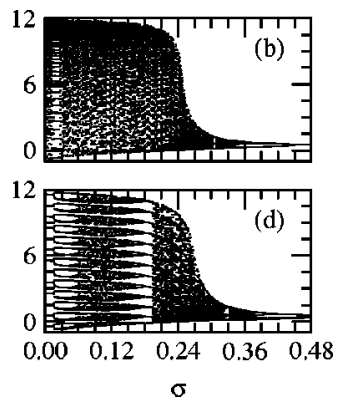

FIG. 8. Structural stability study or the $2 \mathrm{H}$ map as displayed by the bifurcation diagrams of some variations of Eq. (6). (a) Using $R=\frac{1}{3}$ for the continuity condition Eqs. (7) and (8). (b) Leaving $f(x)$ as in Eq. (6) for $x \leqslant \bar{x}$ and using $\sigma / x+1.08 x-1$ for $x>\bar{x}$. Leaving $f(x)$ as in Eq. (6) for $x>\bar{x}$ and using (c) $0.10 /(x-\chi)+18$, or (d) $0.20 /(x-\chi)+12$ for $x \leqslant \bar{x}$.

changes in the attractor size, in formal correspondence with first order and higher order phase transitions. This correspondence breaks down in our case when the derivatives of the width of the attractor are considered, because the infinite divergence proper of second order phase transitions is not observed here in the Hindmarsh-Rose model. An infinite divergence, maintaining the continuous crisis, could be enforced in the map by changing its first branch for a function having an infinite slope at $\bar{x}$. This could be achieved, for example, using the upper arc of an hyperbola with its symmetry axis parallel to the $x$-axis and its vertex in the inflection point $(\bar{x}, \bar{y})$. Other curves, such as the first quadrant of an ellipse with its lower point at $(\bar{x}, \bar{y})$, would have also worked. This has not been done here because this divergence at $\sigma_{C}$ is not observed in the Hindmarsh-Rose model. This discussion, however, suggests that the dynamic behaviors available to the $2 \mathrm{H}$ map could deserve additional independent study from the point of view of theoretical physics and mathematics, beyond the interdisciplinary biophysical approach given to the present article.

\section{DISCUSSION AND CONCLUSIONS}

The observation of a continuous interior crisis in the Hindmarsh-Rose neuron model presented here is relevant both for the theory of nonlinear dynamics and chaos, and for biophysics, particularly for neurobiology.

The study of transitions between different dynamic behaviors in nonlinear systems is an issue of major interest for the theory of nonlinear dynamics and chaos. An important result in this field has been the discovery of different routes to chaos. ${ }^{17,18}$ These are transition phenomena that make chaotic a nonchaotic system when a control parameter is changed, which include period doubling cascades, intermittency, and Hopf bifurcations among others. Less attention, however, has been received by the chaos-chaos transitions in which relevant observables of a chaotic attractor change in a sudden way with the control parameter. In this case, two copies of the same system having very close parameter values may bear very different properties, still being both chaotic. The discontinuous interior crisis ${ }^{16}$ is a well known phenomenon of this kind. In this article, by means of a particular observation, we have proven the existence of a new type of chaos-chaos transition, which bears certain resemblance with the discontinuous interior crisis, and which may, and should not, be confused with it. Moreover, we have presented a simple mechanism which, being based on universal models of chaotic dynamics, possibly gets the essence of this transition not only in the Hindmarsh-Rose model but also in other bursting-spiking systems.

On the other hand, the existence of continuous interior crisis in the Hindmarsh-Rose neuron model has also potential applications in biophysics because it provides a switching mechanism by which a very small change on a system parameter, or on a external input, can toggle the dynamics between two significantly different dynamic behaviors. An example illustrating this occurs in neurobiology, where a major problem is to understand how the nervous system is able to give quick responses given the presence of time delays and noise (see Refs. 14 and 15 and references therein). The present study provides a mechanism which allows rapid switches between different neuronal dynamic behaviors: provided that the system was initially tuned close to the continuous interior crisis, small changes in neuronal input would produce very rapid changes in the pattern of neural spiking. Thus the occurrence of continuous interior crisis in neural models might be relevant because it provides a new mechanism to deal with this problem. This is an alternative to multistable dynamic systems, which is the only other possibility that has been proposed until now. ${ }^{19,20}$

In conclusion, by means of the study of the bursting dynamics of the Hindmarsh-Rose neuron model, a sharp chaos-chaos transition has been identified in which the shape of the attractor changes in an abrupt but continuous way together with other relevant properties of the system. The transition has a well defined critical point and a narrow critical region around it. This transition is different from the well known interior crisis, which is a discontinuous change of size. Because of the formal analogy with phase transitions in condensed matter physics we have proposed to call this new transition a continuous interior crisis. Its mechanism, as shown by means of the introduction of a new onedimensional discrete map, the $2 \mathrm{H}$ map, happens to be a crossover between two different universal forms of chaotic behavior which has to be sharp for a well defined bursting regime to exist. These results have natural applications in biophysics where bursting-spiking chaotic systems are common, and might be relevant in other fields, such as chemistry, where chaos-chaos transitions occur.

\section{ACKNOWLEDGMENT}

This research has been supported by DGI through Project No. BFM2000-0606.

\footnotetext{
${ }^{1}$ F. Rieke, D. Warland, R. de Ruyter van Stevenick, and W. Bialek, Spikes: Exploring the Neural Code (MIT, Cambridge, MA, 1997).

${ }^{2}$ G. Deco and B. Schürman, Phys. Rev. Lett. 97, 4697 (1997).

${ }^{3}$ H. Hasegawa, Phys. Rev. E 61, 718 (2000).

${ }^{4}$ P. Parmanada, C. H. Mena, and G. Baier, Phys. Rev. E 66, 047202 (2002).

${ }^{5}$ C. Morris and H. Lecar, Biophys. J. 35, 193 (1981).

${ }^{6}$ G. Houart, G. Dupont, and A. Goldbeter, Bull. Math. Biol. 61, 507 (1999).

${ }^{7}$ A. M. Zhabotinsky, Chaos 1, 379 (1991).
} 
${ }^{8}$ J. L. Hindmarsh and R. M. Rose, Proc. R. Soc. London, Ser. B 221, 87 (1984).

${ }^{9}$ M. I. Rabinovich, D. I. Abarbanel, R. Huerta, R. Elson, and A. I. Selverston, IEEE Trans. CAS 44, 997 (1997).

${ }^{10}$ W. Wand, Y. Wang, and Z. D. Wang, Phys. Rev. E 57, R2527 (1998).

${ }^{11}$ J. P. Baltanás and J. M. Casado, Phys. Rev. E 65, 041915 (2002).

${ }^{12}$ R. Huerta, M. Bazhenov, and M. I. Rabinovich, Europhys. Lett. 43, 719 (1998).

${ }^{13}$ D. H. He, G. Hu, M. Zhan, and H. P. Lu, Physica D 156, 314 (2001).

${ }^{14}$ J. G. Milton and M. C. Mackey, J. Physiol. (Paris) 94, 489 (2000).
${ }^{15}$ C. Keysers, D.-K. Xiao, P. Földiák, and D. I. Perrett, J. Cogn. Neurosci. 13, 90 (2001)

${ }^{16}$ C. Grebogi, E. Ott, and J. A. Yorke, Phys. Rev. Lett. 48, 1507 (1982).

${ }^{17}$ A. J. Lichtenberg and M. A. Lieberman, Regular and Chaotic Dynamics (Springer-Verlag, New York, 1992).

${ }^{18}$ E. Ott, Chaos in Dynamical Systems (Cambridge University Press, Cambridge, UK, 2002).

${ }^{19}$ J. Foss, A. Longtin, B. Mensour, and J. Milton, Phys. Rev. Lett. 76, 708 (1996).

${ }^{20}$ J. Foss and J. Milton, J. Neurophysiol. 84, 975 (2000). 